\title{
Sustainable Management of Marine Fish Stocks by Means of Sliding Mode Control
}

\author{
Katharina Benz \\ Institute of Product \\ and \\ Process Innovation \\ Leuphana University of Lueneburg \\ Volgershall 1, \\ D-21339 Lueneburg, Germany. \\ Email: katharina.benz@stud.leuphana.de
}

\author{
Claus Rech \\ Institute of Product \\ and \\ Process Innovation \\ Leuphana University of Lueneburg \\ Volgershall 1, \\ D-21339 Lueneburg, Germany. \\ Email: clausrech@gmail.de
}

\author{
Paolo Mercorelli \\ Institute of Product \\ and \\ Process Innovation \\ Leuphana University of Lueneburg \\ Volgershall 1, \\ D-21339 Lueneburg, Germany. \\ Email: mercorelli@uni.leuphana.de
}

\begin{abstract}
This paper deals with a possible approach to controlling marine fish stocks using the prey-predator model described by the Lotka-Volterra equations. The control strategy is conceived using the sliding mode control (SMC) approach which, based on the Lyapunov theorem, offers the possibility to track desired functions, thus guaranteeing the stability of the controlled system. This approach can be used for sustainable management of marine fish stocks: through the developed algorithm, the appropriate number of active fishermen and the suitable period for fishing can be determined. Computer simulations validate the proposed approach.
\end{abstract}

\section{INTRODUCTION AND MOTIVATION}

$\mathbf{M}$ Arine ecosystems provide humanity with a multitude of goods and services, including water quality, flood control and food supply, all of which are critical for human welfare. Since the human population is growing continuously, the demand for these goods and services is also increasing and progressively exerting more pressure on aquatic ecosystems. As many fish species migrate frequently and the oceans are mostly defined as public areas, the definition of clear boundaries and property rights regarding marine resources is rather complicated. As a result, most natural resources exploited by the fishing industry are defined as common-pool resources. This has resulted in many pelagic ecosystems experiencing high levels of depletion and overexploitation [2], with 46 $\%$ of European community fish stocks currently below their minimum biological level (European Environment Agency, [1]). The increasing intensity of human fishing activities in turn diminishes the biodiversity within the affected systems, which is positively correlated with the provision of the goods and services of the ecosystem that are of benefit to the human population, see [5]. Levels of biodiversity have been shown to determine the stability of marine ecosystems and their ability to recover. Consequently, Worm et al. suggest that business as usual in the fishing industry could potentially threaten global food security and water quality, as well as ecosystem resilience, and thus jeopardise present and future generations, see [5]. The observed trend is thus of increasing concern, so the topic of the conservation and restoration of aquatic biodiversity through sustainable fishery management is increasingly visible in scientific and political agendas. The United Nations has included this issue in its sustainable development goals, dedicating goal number 14 to the conservation and sustainable usage of the planet's oceans, seas and marine resources, [4]. The successful implementation of this goal includes the adaptation of sustainable methods to manage marine and coastal ecosystems in order to avoid significant adverse effects, which is indicated by the proportion of national economic zones following ecosystem-based approaches. By 2020, the United Nations aims to regulate destructive fishing activities and end overfishing, alongside implementing a science-based management approach to restore natural fish stocks (United Nations, 2019). In addition, the European Union has conducted several reforms of the Common Fisheries Policy (CFP), establishing different approaches to attempt to bring the situation under control, with the goal of reaching and maintaining a sustainable level of fish in the oceans and in fishermen's nets. As common practice in this field, scientists estimate the existing level of fish stocks within an area and suggest a number of total allowable catches (TACs) to political fishery ministers. In turn, those ministers try to bargain and receive the highest shares for their regions, which often leads to the amount of TACs exceeding the maximum level recommended by scientists, rather than levels being allocated for mutual benefit and optimal conservation purposes. As a result, the methods of the EU are rather unsuccessful for maintaining a sustainable yield of fish and achieving the targets adopted by all member states of the United Nations: as [3] claims, the decision-making process within the catch allocation should be managed by scientists rather than by politicians. One possible approach to enhancing this decision-making process and expanding it based on an independent and objective component, driven by scientific data, is to translate the observed ecosystem into a mathematical model using MATLAB and simulate them with the integrated tool Simulink. Thus, this paper aims to offer a first attempt at exploring how MATLAB and Simulink can be utilised to facilitate the implementation of sustainable management approaches in the fishing industry through strategic policy testing. The software will be used 
to formulate a simple mathematical description of a marine ecosystem based upon the prey-predator system represented in the Lotka-Volterra equations. A number of papers dealing with simulated prey-predator systems have been published previously; however, adaptation of the model to a marine ecosystem including fish stocks and human fishers has not yet been covered. In order to simulate the consequences of various possible policies through different controllers, these have been incorporated into the code to eventually reach and maintain a certain setpoint equal to the maximum sustainable yield of fish. In terms of the proposed control technique, sliding mode control (SMC) is taken as one of the first possible approaches. In fact, the controllers obtained by an SMC approach show robust properties with respect to parameter uncertainties, as well with respect to more general dynamic uncertainties and to unknown signals. Another application for which SMC has suitable qualities is the field of fault-tolerant control (FTC). In this area, due to intrinsic robustness, SMC models are able to overcome faults and uncertainties. The paper is organised in the following way. In Section II the Lotka-Volterra model is presented. Section III is devoted to the control design performed using SMC. Section IV presents the obtained results and the paper ends with the conclusions drawn.

\section{MODEL DESIGN}

The designed model is inspired by the ecological concept of the prey and predator relationship. This concept was formulated by Lotka and Volterra, and is based upon different mathematical theorems.

\section{A. Lotka-Volterra equations}

The assumptions of Lotka and Volterra are taken as a basis to describe the relationship between natural fish stocks and the fishing activities of humans. Lotka and Volterra first describe the population dynamics of two species in a prey and predator relationship through two first-order nonlinear differential equations, as follows:

$$
\begin{aligned}
& \frac{d x(t)}{d t}=\alpha x(t)-\beta x(t) y(t), \\
& \frac{d y(t)}{d t}=\delta x(t) y(t)-\gamma y(t),
\end{aligned}
$$

where $x(t)$ represents the number of prey and $y(t)$ represents the number of predators. $\frac{d x(t)}{d t}$ and $\frac{d y(t)}{d t}$ represent the growth rates of the populations based on the respective changes within their population sizes over time, which is denoted by the term $t . \alpha, \beta, \delta, \gamma$ are positive real parameters and describe the interaction between the two populations. The expression (1) represents the dynamics of the prey population, which are calculated by subtracting the rate of predation from the population's intrinsic growth rate. Since it is assumed that the prey has an unlimited food supply, its population grows exponentially if the population of predators and the rate of predation equal zero, which is expressed by the term $\alpha x(t)$. In turn, the rate of predation upon the prey is assumed to be proportional to $\beta x(t) y(t)$. Thus, if either $x(t)$ or $y(t)$ equals zero, there is no predation.

Equation (2) describes the dynamics of the predator population, which are determined by the rate at which it consumes the prey population, minus its intrinsic death rate. Since the growth rate of the predator population does not necessarily equal the rate of predation of the prey, it is expressed by $\delta x(t) y(t)$, which is similar but not equal to the term representing the rate of predation in Eq. (1). In this equation, $\gamma y(t)$ denotes the loss rate of the predator population due to natural death or emigration. This results in an exponential decay if there is no prey available to be consumed. Since the main objective of designing this new approach is to achieve and maintain sustainable levels of fish stocks and harvests alike, an equilibrium point between the two populations is intended. This point is reached if:

$$
\begin{aligned}
& \frac{d x(t)}{d t}=0, \\
& \frac{d y(t)}{d t}=0 .
\end{aligned}
$$

As a result, putting the corresponding equations also equal zero, wherefore one has:

$$
\begin{aligned}
& 0=\alpha x(t)-\beta x(t) y(t), \\
& 0=\delta x(t) y(t)-\gamma y(t) .
\end{aligned}
$$

These equations yield two different solutions. One solution states that both populations become extinct:

$$
x(t)=0, \quad y(t)=0 .
$$

Given the second solution, a fixed point can be achieved at which both populations sustain their current non-zero numbers, depending on the settings of the four parameters $\alpha, \beta, \delta, \gamma$. This yields:

$$
\begin{aligned}
& y(t)=\frac{\alpha}{\beta}, \\
& x(t)=\frac{\gamma}{\delta} .
\end{aligned}
$$

\section{SLIDING MODE CONTROL}

As the goal of the simulation is to realise and establish sustainable fishing activities in order to ensure the continuity of both marine ecosystems and the human species, the current situation of overfishing and ocean depletion has to be stopped and managed in a way that enables fish stocks to recover. Therefore, the error between the desired setpoint, being the equilibrium point of the fishery system, and the actual value, represented by the current level of fish, has to be ascertained, harmonised and stabilised. This is explored through application of the Lyapunov theorem. With zero being the intended value for $\dot{x}(t)=f(x, u, t)$, the theorem defines that if:

$$
\begin{gathered}
V(x(t))>0, \forall x(t), \\
V(0)=0,
\end{gathered}
$$

the function is positive and if:

$$
\dot{V}(x(t))<0, \forall x(t)
$$


and one has:

$$
\dot{x}(t)=f(x, u, t),
$$

then $x(t)=0$ is an asymptomatic stabile point for function $\dot{x}(t)=f(x, u, t)$.

In order to reduce the error and harmonise the actual value of fish with the desired value of fish associated with a sustainable population size, an SMC is used as follows:

$$
S(t)=\left(x_{d}(t)-x(t)\right)+k_{s} \int_{0}^{t}\left(x_{d}(z)-x(z)\right) d_{z},
$$

where $k_{s}$ is a parameter to be designed. Since the V-function is a positive-define function of $x(t)$, it can be employed in the function above. Therefore, one gets:

$$
V(S(t))=\frac{1}{2} S^{2}(t)
$$

Thereupon, the function is differentiated, which yields:

$$
\begin{gathered}
\dot{V}(S(t))=\frac{1}{2} 2 S(t) \dot{S}(t) \\
=S(t)\left[\left(\dot{x}_{d}(t)-\dot{x}(t)\right)+k_{s}\left(x_{d}(t)-x(t)\right)\right] \\
=S(t)\left[\dot{x}_{d}(t)-(\alpha x(t)-\beta x(t) y(t))+k_{s}\left(x_{d}(t)-x(t)\right)\right],
\end{gathered}
$$

if: $y(t)=y_{e q}(t)=$

$$
\frac{-\dot{x}_{d}(t)+\alpha x(t)-k_{s}\left(x_{d}(t)-x(t)\right)}{\beta x(t)},
$$

then $\dot{V}(S(t))=0$ and if:

$$
y(t)=y_{e q}(t)-\frac{\eta \mathrm{s} g n(S(t))}{\beta x(t)},
$$

with

$$
\operatorname{sgn}(S(t))=\left\{\begin{array}{l}
1 \text { if } S(t)>0 \\
0 \text { if } S(t)=0 \\
-1 \text { if } S(t)<0
\end{array}\right.
$$

then, if $\eta>0$ :

$$
\begin{aligned}
\dot{V}(S(t))=S(t) & {[-\eta \operatorname{sgn}(S(t))] } \\
& =-\eta S(t) \operatorname{sgn}(S(t))=-\eta|S(t)|<0 .
\end{aligned}
$$

In order to accelerate the process and reach the desired value more quickly, term $\lambda S(t)$, with $\lambda>0$, can be included in the equation. The resulting control law is as follows:

$$
y(t)=y_{e q}(t)-\frac{\eta \mathrm{s} g n(S(t))}{\beta x(t)}-\frac{\lambda S(t)}{\beta x(t)} .
$$

\section{A. Euler Method}

Since the system in question has a relatively slow dynamics, it is not intended to measure its state second-by-second, but rather on a monthly basis. Therefore, the equation is discretised according to the Forward Euler method, where $k$ represents the known counting integer variable, which yields:

$$
\begin{gathered}
\dot{x}(t)=\frac{x(k)-x(k-1)}{T_{s}} \\
=\alpha x(k-1)-\beta x(k-1) y(k-1)
\end{gathered}
$$

$$
\begin{aligned}
\rightarrow x(k)=x(k-1)+T_{s}( & \alpha x(k-1) \\
& -\beta x(k-1) y(k-1)) .
\end{aligned}
$$

At this point, the respective equations are integrated into Matlab. With the number of predators and respectively the number of fishermen represented by $y(t)$, being the leverage point to control the level of fish stocks in the regarded aquatic ecosystem, Eq. (23) represents one of the main equations in the SMC. Since the goal of the applied controller is to harmonise the desired and actual amounts of fish, measured in kilogram biomass, the desired amount of fish (denoted by $x_{d}(t)$ ) and the actual amount of fish (represented by $x(t)$ ) are the two main data inputs for the equation. Eq. (23) represents the main equation within the SMC strategy.

\section{Simulation RESUlts}

In order to test the designed model it is assumed that a sustainable level of fish stocks is reached at a minimum of $10,000 \mathrm{~kg}$ of fish. The goal is then to test how the attendance of fishermen affects the dynamics of the prey population and how a meaningful policy designed to regulate the activities of the fishermen could be framed. Figure 1 shows the number of fishermen in a system that is not restricted by political regulations. The line graph shows the development of the number of fishermen over a period of 60 months. In the absence of political regulations, the number of fishermen immediately increases to 1,000 and remains stable over the entire period of time. The line graph depicted in Fig. 2

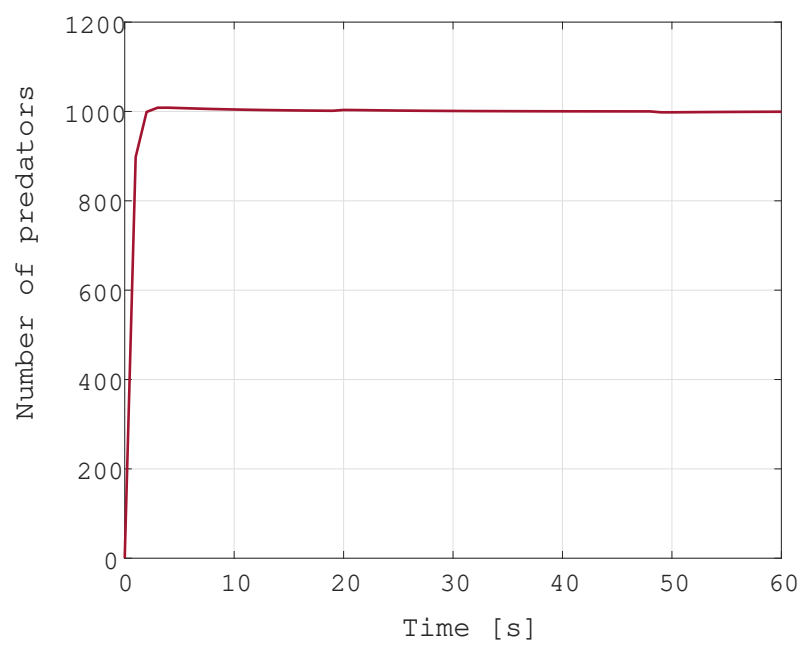

Fig. 1. Number of predators without regulation

shows the corresponding dynamics of the fish population over a period of 60 months, given the same situation that no political regulation of fishing activities exists. In this scenario the amount of fish peaks at $11,000 \mathrm{~kg}$ after approximately three months and stabilises at the desired amount of $10,000 \mathrm{~kg}$ after 60 months. In order to test how a political regulation regarding the number of active fishermen affects the system, a hypothetical regulation has been assumed demanding that all 


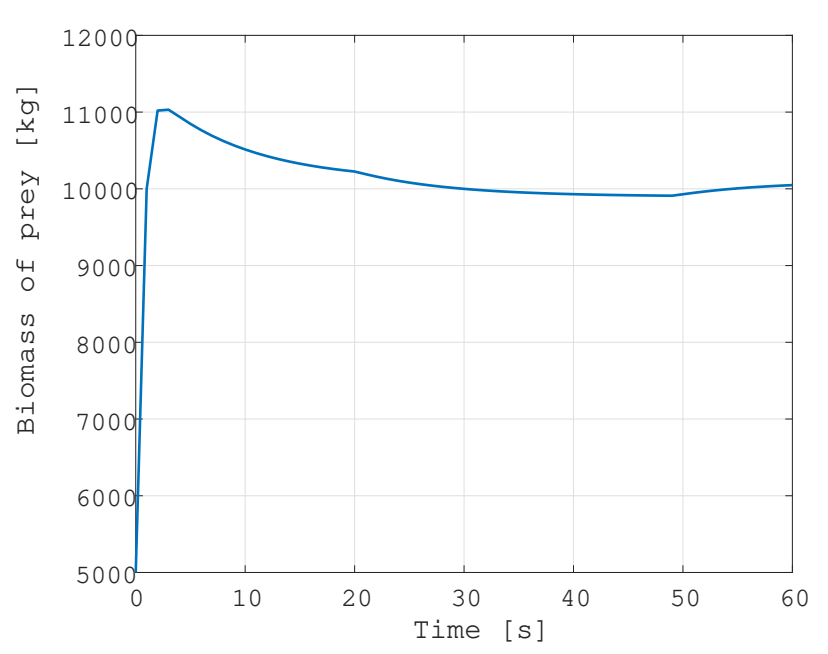

Fig. 2. Biomass of prey without regulation

fishing activities are prohibited between the $5^{\text {th }}$ and the $8^{\text {th }}$ month of the period in question. This regulation is realised through an if-clause in the $\mathrm{m}$-file of Matlab, as follows: if $((T<5) \mid(T>8))$

$$
y(t)=y_{e q}(t)-\frac{\eta \operatorname{s} g n(S(t))}{\beta x(t)} .
$$

As a result, the number of fishermen depicted in Fig. 3 rises to 1,000 and remains at that level until it drops to 0 at the five-month mark. It then remains at 0 until the $8^{\text {th }}$ month and temporarily increases to 1,100 after this point. Subsequently, the number slowly decreases again until it returns to a level of 1,000 after 60 months. The consequences of the regulation

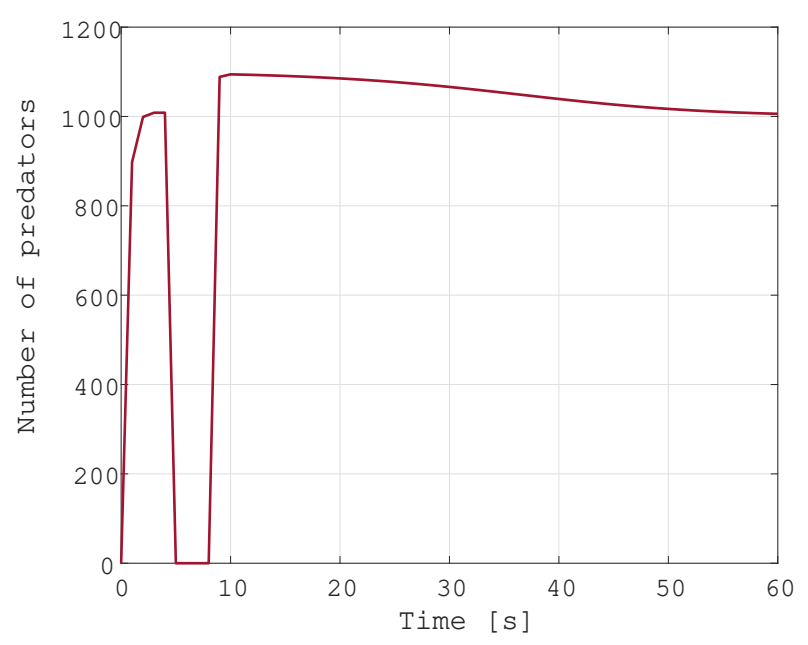

Fig. 3. Number of predators with regulation

regarding the level of fish stocks in kilogram biomass is depicted in Fig. 4. At the beginning of the time period in question, when the number of fishermen is high, the fish biomass level is at $10,000 \mathrm{~kg}$. As soon as the regulation takes effect, the fish biomass increases exponentially, peaking at $17,500 \mathrm{~kg}$ at eight months. Since the fishermen resume their activities from the $8^{t h}$ month onwards, the biomass level decreases again, stabilising at the desired level of $10,000 \mathrm{~kg}$ after 60 months. The results show that the designed model is

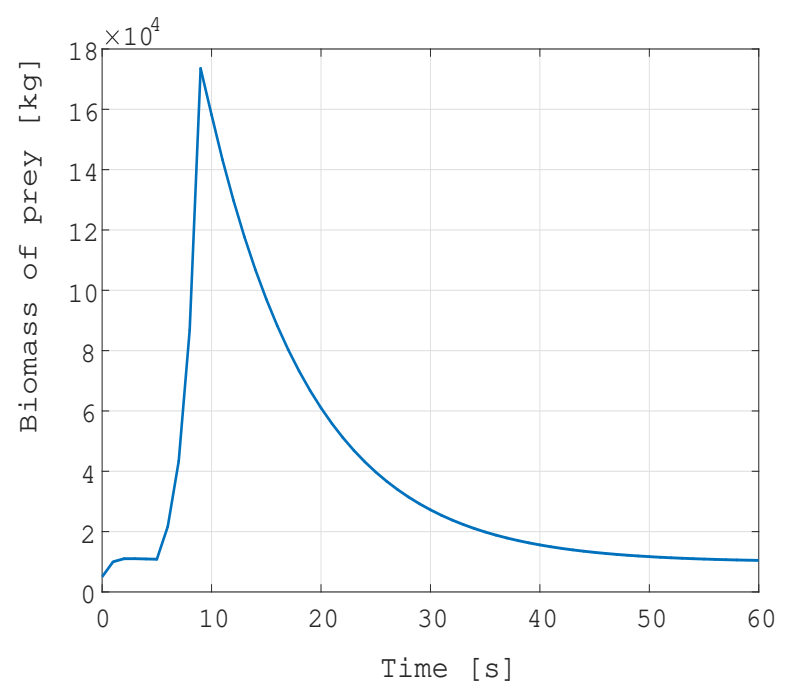

Fig. 4. Biomass of prey with regulation

indeed sensitive to regulatory changes, and that it is able to depict the dynamics of the interdependent populations.

\section{CONCLUSION AND FUTURE RESEARCH}

Since the implementation of a regulating if-clause in the $\mathrm{m}$-file yields a reasonable result, the model seems to work and to be appropriate for policy testing in the fishing industry. However, further research will be necessary in order to construct more complex models, and thus more realistic ones, by including additional variables that may influence the system. In addition, appropriate measurements must be taken and the values within the models must be adapted accordingly in order to obtain realistic and meaningful results.

\section{ACKNOWLEDGMENT}

This work was realised within the lectures for the Complementary Studies course at Leuphana University of Lueneburg during the winter semester 2018-2019.

\section{REFERENCES}

[1] European Environment Agency (EEA) (2010). EU 2010 biodiversity baseline.

[2] Gordon, H. S. The economic theory of a common-property resource: the fishery. The Journal of Political Economy, Vol. 62, No. 2 (Apr. 1954), pp. 124-142

[3] Leary, B. C., Smart, J. C., Neale, F. C., Hawkins, J. P., Newman, S., Milman, A. C., \& Roberts, C. M. (2011) Fisheries mismanagement. Marine Pollution Bulletin, 62 (12), pp. 2642-2648.

[4] United Nations (2019). Sustainable Development Goal 14. https://sustainabledevelopment.un.org/sdg 14 (20.03.2019).

[5] Worm, B., Barbier, E. B., Beaumont, N., Duffy, J. E., Folke, C., Halpern, B. S., ... \& Sala, E. (2006). Impacts of biodiversity loss on ocean ecosystem services. Science, 314 (5800), pp. 787-790. 